Communications in Physics, Vol.21, No. 3 (2011), pp. 277-288

\title{
FREE ENERGY DENSITY FOR GAUGE BACKGROUND FIELDS AT FINITE TEMPERATURE
}

\author{
PHAN HONG LIEN \\ Military Academy of Technology
}

\begin{abstract}
The effective action and background field method have been applied to investigate free energy density for non-Abelian gauge theory at finite temperature, in which quantum corrections are included and certain symmetries of generating functional are restored. Renormalization is also considered for the gauge field. We give result for the one loop free energy density of gauge theory at high temperature and non-zero chemical potential, correcting a result previously at zero temperature and density. Some results are extended up to two loops.
\end{abstract}

\section{INTRODUCTION}

The finite temperature effective action and its thermal potential is known as a method which provides a general approximation beyond one loop and higher free energy density, in the perturbative as well as non-perturbative sector [1]. In particular, it plays an important role in the investigation of cosmological phase transition [2] and non-equilibrium phenomena [3]. However, the effective potential of gauge theories may fail to be gauge because it is against gauge transformation and generally does depend on the $\xi$-gauge $[3$, 4]. Therefore, it is worth to mention that the background field method allows one to fix a gauge, thereby compute quantum effects without losing explicit gauge invariance $[5,6]$.

Our main aim is to present in detail the effective action at high temperature for general non-Abelian theory by background gauge field method. In this connection, it is possible to consider our work as being complementary to result previously at zero temperature [6].

This paper is organized as follows. In Sec. II, the background field method is presented for general fermion-boson interacting system. Section III is devoted to effective potential at finite temperature for background fields. In Sec. IV the dimensional regularization at finite temperature is presented for some divergent integrals. We obtained the result for one loop free energy density and renormalization of the gauge field. The discussion and conclusion are given in Sec. V.

\section{FORMALISM}

\section{II.1. Background field method}

Let $I\left(\bar{\Psi}, \Psi, \Phi, A_{\mu}\right)$ be the action of fermion - boson interacting system, where $\bar{\Psi}, \Psi$ are multiplet of fermion fields, $\Phi_{i}(i=1,2 \ldots n)$ are components of the scalar fields, $A_{\mu}$ - 
gauge fields and $\omega, \omega^{*}$ - ghost fields.

$$
I=\int d x L_{0}(x)
$$

where Lagrangian density reads

$$
\begin{aligned}
L_{0}= & -\frac{1}{4} F_{\mu \nu}^{a} F^{a \mu \nu}+\bar{\Psi}\left(i \gamma^{\mu} D_{\mu}-G_{i} \Phi_{i}\right) \Psi \\
& +\left[\left(D_{\mu}-i \mu \delta_{\mu 0}\right) \Phi_{i}\right]^{+}\left[\left(D^{\mu}-i \mu \delta^{\mu 0}\right) \Phi_{i}\right]-m^{2} \Phi_{i}^{+} \Phi_{i}-\lambda\left(\Phi_{i}^{+} \Phi_{i}\right)^{2} \\
& -\frac{1}{2 \xi}\left(\partial^{\mu} A_{\mu}^{a}\right)^{2}-\partial_{\mu} \omega_{a}^{*} \partial^{\mu} \omega_{a}+f_{a b c}\left(\partial_{\mu} \omega_{a}^{*}\right) A_{\mu}^{b} \omega^{c} .
\end{aligned}
$$

Here $\mu$ is chemical potential, $G_{i}$ and $\lambda$ are coupling constants, $\lambda>0$

$$
\begin{aligned}
D_{\mu} & \equiv \partial_{\mu}-i T^{a} A_{\mu}^{a}, \\
F_{\mu \nu}^{a} & \equiv \partial_{\mu} A_{\nu}^{a}-\partial_{\nu} A_{\mu}^{a}+f_{a b c} A_{\mu}^{b} A_{\nu}^{c},
\end{aligned}
$$

where $T_{a}$ are group generators, $f_{a b c}$ are structure constants which satisfy Lie algebra

$$
\begin{aligned}
& f_{a b c} f_{d b c}=g^{2} C_{A} \delta_{a d} \\
& \operatorname{Tr} T_{a} T_{b}=g^{2} C_{F} \delta_{a b}
\end{aligned}
$$

with $C_{A}$ is numerical constant of gauge group, $C_{A}=N$ for $S U(N), C_{F}$ is representation of this group.

It is well known, the Lagrangian (1) is invariant under non-abelian gauge transformation.

$$
\begin{aligned}
\Psi(x) & \rightarrow U \Psi(x), \\
A_{\mu}(x) & \rightarrow U A_{\mu}(x) U^{+}+\frac{i}{g}\left(\partial_{\mu} U\right) U^{+}, \\
F_{\mu \nu}(x) & \rightarrow U F_{\mu \nu}(x) U^{+}
\end{aligned}
$$

where $U$ is unita transformation of gauge parameters

$$
U=e^{-i \theta(x)}, \theta(x)=\theta^{a}(x) T^{a}, A_{\mu}(x)=A_{\mu}^{a}(x) T^{a} .
$$

In the generalized $\xi$ - gauge, the Lagrangian (1) is also renormalizable (if the matter Lagrangian part is).

In the non - abelian theory, explicit gauge invariance is normally lost when quantum correction are included. The background field method allows us to fix gauge, hence investigate quantum effects, so that certain symmetries of generating functional are restored.

The fields are shifted by

$$
\begin{aligned}
& A_{\mu} \rightarrow \mathbf{A}_{\mu}+A_{\mu}^{\prime} ; \quad\left\langle 0\left|\mathbf{A}_{\mu}\right| 0\right\rangle=\text { const }, \quad\left\langle 0\left|A_{\mu}^{\prime}\right| 0\right\rangle=0, \\
& \Phi_{i} \rightarrow \boldsymbol{\Phi}_{i}+\Phi_{i}^{\prime} ; \quad\left\langle 0\left|\boldsymbol{\Phi}_{i}\right| 0\right\rangle=\phi_{0 i}, \quad\left\langle 0\left|\Phi_{i}^{\prime}\right| 0\right\rangle=0, \\
& \Psi \rightarrow \boldsymbol{\Psi}+\Psi^{\prime} ; \quad\langle 0|\boldsymbol{\Psi}| 0\rangle=\left\langle 0\left|\boldsymbol{\Psi}^{\prime}\right| 0\right\rangle=0, \\
& \omega_{a} \rightarrow \boldsymbol{\omega}_{a}+\omega_{a}^{\prime} ; \quad\left\langle 0\left|\boldsymbol{\omega}_{a}\right| 0\right\rangle=\left\langle 0\left|\omega_{a}^{\prime}\right| 0\right\rangle=0,
\end{aligned}
$$

where $\mathbf{A}_{\mu}, \boldsymbol{\Phi}, \boldsymbol{\Psi}, \boldsymbol{\omega}_{a}$ are the background fields, and $A_{\mu}^{\prime}, \Phi^{\prime}, \Psi^{\prime}, \omega_{a}^{\prime}$ are the quantum fields, which are variables of integration in the functional integral. The gauge is chosen (the 
background field) which breaks the gauge invariance in terms of the $\mathbf{A}_{\mu}, \boldsymbol{\Phi}, \mathbf{\Psi}, \boldsymbol{\omega}$ fields. Background field gauge is assured by coupling external sources only to the $A_{\mu}^{\prime}, \Psi^{\prime}, \Phi^{\prime}, \omega^{\prime}$ fields. Thus, quantum calculations can be performed, yet explicit gauge invariance in the background field variable is not lost.

It is shown [6] that the original gauge transformation is equivalent to formal combined transformations

$$
\begin{aligned}
\delta \mathbf{A}_{\mu}^{a} & =\partial_{\mu} \theta^{a}-f_{a b c} \theta^{b} \mathbf{A}_{\mu}^{c}, & \delta A_{\mu}^{\prime a} & =-f_{a b c} \theta^{b} A_{\mu}^{\prime c}, \\
\delta \boldsymbol{\Psi} & =i \theta^{a} T^{a} \Psi, & \delta \Psi^{\prime} & =i \theta^{a} T^{a} \Psi^{\prime}, \\
\delta \boldsymbol{\omega}_{a} & =-f_{a b c} \theta^{b} \omega_{c}, & \delta \omega_{a}^{\prime} & =-f_{a b c} \theta^{b} \omega_{c}^{\prime}
\end{aligned}
$$

so that

$$
\begin{aligned}
\delta\left(\mathbf{A}_{\mu}^{a}+A_{\mu}^{\prime a}\right) & =\partial_{\mu} \theta^{a}-f_{a b c} \theta^{b}\left(\mathbf{A}_{\mu}^{c}+A_{\mu}^{\prime c}\right), \\
\delta\left(\boldsymbol{\Psi}+\Psi^{\prime}\right) & =i \theta^{a} T^{a}\left(\boldsymbol{\Psi}+\Psi^{\prime}\right), \\
\delta\left(\boldsymbol{\omega}_{a}+\omega_{a}^{\prime}\right) & =-f_{a b c} \theta^{b}\left(\boldsymbol{\omega}_{c}+\omega_{c}^{\prime}\right)
\end{aligned}
$$

If we choose the gauge fixing function $F^{a}$ which transforms as a background covariant derivation

$$
F^{a}=\bar{D}_{\mu} A_{\mu}^{\prime a},
$$

where $\bar{D}_{\mu} \varphi_{a}=\partial_{\mu} \varphi_{a}+f_{a b c} A_{b \mu} \varphi_{c}$ for any field $\varphi_{a}$, then

$$
\delta F^{a}=-f_{a b c} \theta^{b} F^{c} ; \quad \delta\left(F^{q} F^{a}\right)=0 .
$$

In the scalar sector of non-Abelian theory, the Lagrangian part

$$
L_{B}=\left(D_{\mu} \boldsymbol{\Phi}_{i}\right)^{+}\left(D^{\mu} \boldsymbol{\Phi}_{i}\right)+i \mu\left[\boldsymbol{\Phi}_{i}^{+}\left(D_{0} \boldsymbol{\Phi}_{i}\right)-\left(D_{0} \boldsymbol{\Phi}_{i}\right)^{+} \boldsymbol{\Phi}_{i}\right]+\left(\mu^{2}-m^{2}\right) \Phi_{i}^{+} \Phi_{i}-\lambda\left(\Phi_{i}^{+} \Phi_{i}\right)^{2}
$$

is invariant under the local transformation

$$
\Phi_{i}(x) \longrightarrow e^{-i \theta(x)} \Phi_{i}(x)
$$

It leads to a new ground state and the shift $\Phi_{i}(x)$ by real fields $\Phi_{i}^{\prime}(x)$, i.e

$$
\Phi_{0 i}=\left\langle\Phi_{i}\right\rangle=\sqrt{\frac{\mu^{2}-m^{2}}{2 \lambda}}=\frac{v}{\sqrt{2}} \longrightarrow \frac{1}{\sqrt{2}}\left(v+\Phi_{i}^{\prime}(x)\right) .
$$

Hence symmetry is broken if $\mu^{2}>m^{2}$.

Since the potential only depends on $\left[v+\Phi_{i}^{\prime}(x)\right]^{2}$, it is clearly that $\theta(x)$ are massless fields (Goldstone bosons), i.e $\langle\theta(x)\rangle=0$. In general, Goldstone bosons don't appear in spectrum of theory in which the symmetry is local.

The formal combined transformation (10) - (12) leave invariance of the modified complete Lagrangian

$$
L_{\text {mod }}=L_{A F}+L_{B}+L_{g f}+L_{g h o s t}
$$

where

$$
\begin{aligned}
L_{A F}= & -\frac{1}{4}\left(F_{\mu \nu}^{a}-\bar{D}_{\mu} A_{\nu}^{\prime a}-\bar{D}_{\nu} A_{\mu}^{\prime a}+f_{a b c} A_{\mu}^{\prime b} A_{\nu}^{\prime c}\right)^{2} \\
& -\Psi^{\prime}\left[i \gamma^{\mu}\left(\bar{D}_{\mu}-i T^{a} A_{\mu}^{\prime a}\right)+M\right] \Psi^{\prime}-g_{i} \bar{\Psi}^{\prime} \Phi_{i}^{\prime} \Psi^{\prime} .
\end{aligned}
$$


Here $g_{i}=\sqrt{2} G_{i}, M=g_{i} v$ is the mass of fermion in the presence of a background field $\boldsymbol{\Phi}_{i}$.

$$
\begin{aligned}
L_{B}= & \frac{1}{2} \bar{D}_{\mu} \Phi_{i}^{\prime} \bar{D}^{\mu} \Phi_{i}^{\prime}+\frac{1}{2} M_{i j}^{2} \Phi_{i}^{\prime} \Phi_{j}^{\prime} \\
& +\frac{1}{2} M_{a b}^{2} A_{\mu}^{\prime a} A_{\mu}^{\prime b}+v \Phi_{i}^{\prime} A_{\mu}^{\prime a} A_{\mu}^{\prime b} T^{a} T^{b}-\lambda v \Phi_{i}^{\prime 3}-\frac{\lambda}{4} \Phi_{i}^{\prime 4} .
\end{aligned}
$$

with $M_{a b}^{2}=g^{2} v^{2} T_{a} T_{b}$ is the mass of vector boson, $M_{i j}^{2}=\delta_{i j}\left(\mu^{2}-m^{2}\right)-\frac{\lambda}{2} \Phi_{i}^{\prime} \Phi_{j}^{\prime}$ is the diagonal mass matrices of scalar bosons.

$$
\begin{aligned}
L_{g f} & =-\frac{1}{2 \xi} F^{a} F^{a}=-\frac{1}{2 \xi}\left(\bar{D}_{\mu} A_{\mu}^{\prime a}\right)^{2}, \\
L_{g h o s t} & =-\left(\bar{D}_{\mu} \omega_{a}^{\prime}\right)\left(\bar{D}^{\mu} \omega_{a}^{\prime}-f_{a b c} \omega_{b}^{\prime} A_{c}^{\prime \mu}\right),
\end{aligned}
$$

where

$$
\begin{aligned}
\bar{D}_{\mu} \Psi^{\prime} & =\partial_{\mu} \Psi^{\prime}-i T^{a} \mathbf{A}_{\mu}^{a} \Psi^{\prime}, \\
\bar{D}_{\mu} \Phi_{i}^{\prime} & =\partial_{\mu} \Phi_{i}^{\prime}-i T^{a} \mathbf{A}_{\mu}^{a} \Phi_{i}^{\prime}, \\
\bar{D}_{\mu} A_{\nu}^{\prime a} & =\partial_{\mu} A_{\nu}^{\prime a}+f_{a b c} \mathbf{A}_{\mu}^{b} A_{\nu}^{\prime c} \\
\bar{D}_{\mu} \omega_{a} & =\partial_{\mu} \omega_{a}+f_{a b c} \mathbf{A}_{b \mu} \omega_{c}^{\prime}, \\
\bar{D}_{\mu} \omega_{a}^{*} & =\partial_{\mu} \omega_{a}^{*}+f_{a b c} \mathbf{A}_{b \mu} \omega_{c}^{\prime *}
\end{aligned}
$$

\section{II.2. Renormalization}

The formal gauge invariance impose the constrains on divergence occurred in the theory, but the background gauge transformations (10) - (12) conserver gauge invariance in path integration.

Adding to the Lagrangian (1) a counterterm

$$
\begin{aligned}
\delta L= & -\frac{1}{4} L_{A} F_{\mu \nu}^{a} F^{a \mu \nu}+L_{\Psi} \overline{\mathbf{\Psi}} \gamma^{\mu} D_{\mu} \boldsymbol{\Psi}-L_{i} G_{i} \overline{\mathbf{\Psi}} \Phi_{i} \boldsymbol{\Psi} \\
& +L_{\Phi}\left[\left(D_{\mu}^{R}-i \mu \delta_{\mu 0}\right) \Phi^{R}\right]^{+}\left[\left(D^{\mu R}-i \mu \delta^{\mu 0}\right) \Phi^{R}\right] \\
& -L_{m} m^{2} \Phi_{i}^{+} \Phi_{i}-L_{\lambda} \lambda\left(\Phi_{i}^{+} \Phi_{i}\right)^{2}-L_{\omega}\left(\bar{D}_{\mu} \omega_{a}^{*}\right)\left(D^{\mu} \omega_{a}\right)
\end{aligned}
$$

so that renormalized Lagrangian takes the form

$$
\begin{aligned}
L^{R}=L_{0}+\delta L= & -\frac{1}{4} F_{\mu \nu}^{a R} F^{a \mu \nu R}-\overline{\mathbf{\Psi}}^{R} \gamma^{\mu} D_{\mu} \boldsymbol{\Psi}^{R}-g_{i}^{R} \overline{\mathbf{\Psi}}^{R} \Phi_{i}^{R} \mathbf{\Psi}^{R} \\
& +\left(D_{\mu}^{R} \Phi_{i}^{R}\right)^{+}\left(D^{\mu R} \Phi_{i}^{R}\right)+i \mu\left(\Phi_{i}^{+R} \partial_{0} \Phi_{i}^{R}-\Phi_{i}^{R} \partial_{0} \Phi_{i}^{+R}\right) \\
& +\left(\mu^{2}-m_{R}^{2}\right) \Phi_{i}^{+R} \Phi_{i}^{R}-\lambda_{R}\left(\Phi_{i}^{+R} \Phi_{i}^{R}\right)^{2}-\frac{1}{2 \xi}\left(D_{\mu}^{R} A_{\mu}^{0 R}\right)^{2} \\
& -\left(D_{\mu}^{R} \omega_{a}^{* R}\right)\left(D^{\mu R} \omega_{a}^{R}\right)
\end{aligned}
$$


Hence, its easily to define the renormalized fields

$$
\begin{aligned}
A_{\mu}^{a R} & =\sqrt{1+L_{A}} A_{\mu}^{a} & \Phi_{i}^{R} & =\sqrt{1+L_{\Phi}} \Phi_{i} \\
\Psi^{R} & =\sqrt{1+L_{\Psi}} \Psi & v^{R} & =\sqrt{1+L_{\Phi}} v \\
\omega_{a}^{R} & =\sqrt{1+L_{\omega}} \omega_{a} & \omega_{a}^{* R} & =\sqrt{1+L_{\omega}} \omega_{a}^{*}
\end{aligned}
$$

and the renormalized masses, the background field strength tensor

$$
\begin{aligned}
& M^{R}=G_{i}^{R} v^{R} ; \quad m_{R}^{2}=m^{2} \frac{\left(1+L_{m}\right)}{1+L_{\Phi}}, \\
& F_{\mu \nu}^{a R}=\partial_{\mu} A_{\nu}^{a R}-\partial_{\nu} A_{\mu}^{a R}+f_{a b c}^{R} A_{\mu}^{b R} A_{\nu}^{c R} .
\end{aligned}
$$

The covariant derivation are taken in renormalized sense

$$
\begin{aligned}
D_{\mu}^{R} \Psi^{R} & =\partial_{\mu} \Psi^{R}-i T_{a}^{R} A_{\mu}^{a R} \Psi^{R} \\
D_{\mu}^{R} \Phi_{i}^{R} & =\partial_{\mu} \Phi_{i}^{R}-i T_{a}^{R} A_{\mu}^{a R} \Phi_{i}^{R} \\
D_{\mu}^{R} A_{\nu}^{a R} & =\partial_{\mu} A_{\nu}^{a R}+f_{a b c}^{R} A_{\mu}^{b R} A_{\nu}^{c R} \\
D_{\mu}^{R} \omega_{a}^{R} & =\partial_{\mu} \omega_{a}^{R}+f_{a b c}^{R} A_{b \mu}^{R} \omega_{c}^{R} \\
D_{\mu}^{R} \omega_{a}^{* R} & =\partial_{\mu} \omega_{a}^{* R}+f_{a b c}^{R} A_{b \mu}^{R} \omega_{c}^{* R} .
\end{aligned}
$$

The renormalized structure constants and group generators are determined by

$$
\begin{aligned}
f_{a b c}^{R} & =\left(1+L_{A}\right)^{-1 / 2} f_{a b c}, \\
T_{a}^{R} & =\left(1+L_{A}\right)^{-1 / 2} T_{a},
\end{aligned}
$$

and the coupling constants are renormalized by

$$
\begin{aligned}
\lambda^{R} & =\lambda\left(1+L_{\lambda}\right)\left(1+L_{A}\right)^{-2}, \\
G_{i}^{R} & =g_{i}\left(1+L_{i}\right)\left(1+L_{\Psi}\right)^{-1}\left(1+L_{\Phi}\right)^{-1 / 2} \\
g^{R} & =g\left(1+L_{A}\right)^{-1 / 2} .
\end{aligned}
$$

\section{THE EFFECTIVE ACTION FOR BACKGROUND FIELDS AT FINITE TEMPERATURE}

The generating functional for the background fields is defined by

$$
\begin{aligned}
Z_{\beta} & {\left[\eta, \bar{\eta}, J_{i}, J_{\mu}, J_{a}, J_{b}^{*}\right] \equiv \exp i W_{\beta}\left[\eta, \bar{\eta}, J_{i}, J_{\mu}, J_{a}, J_{b}^{*}\right] } \\
& =\int[d \overline{\mathbf{\Psi}}][d \boldsymbol{\Psi}][d \boldsymbol{\Phi}][d \boldsymbol{A}][d \boldsymbol{\omega}]\left[d \boldsymbol{\omega}^{*}\right] \exp i\left\{\int d x L_{\text {mod }}(x)+\mu \overline{\mathbf{\Psi}} \gamma_{0} \boldsymbol{\Psi}\right\} \\
& +\int d x\left[\bar{\eta} \boldsymbol{\Psi}+\overline{\mathbf{\Psi}} \eta+J_{i} \boldsymbol{\Phi}_{i}+J^{\mu} \boldsymbol{A}_{\mu}+J_{a}^{*} \boldsymbol{\omega}_{a}+J_{b} \boldsymbol{\omega}_{b}^{*}\right] .
\end{aligned}
$$

The integration has to be performed over antiperiodic Grassman fields

$$
\Psi(0, \mathbf{x})=-\Psi(\beta, \mathbf{x})
$$

and periodic bosonic fields

$$
\mathbf{B}(0, \mathbf{x})=B(\beta, \mathbf{x}) \text { with } \mathbf{B}=\left(\mathbf{\Phi}, \mathbf{A}_{\mu}\right),
$$


where $\beta=\frac{1}{k T}$, we set Boltzman constant $k=1$.

If we complete the background gauge transformation with transformation for the external sources

$$
\begin{aligned}
\delta \eta & =i \theta^{a} T^{a} \eta & & \delta J_{\mu}^{a}=f_{a b c} \theta^{b} J_{\mu}^{c}, \\
\delta \bar{\eta} & =i \bar{\eta} \theta^{a} T^{a} & & \delta J_{a}=f_{a b c} \theta^{b} J^{c}, \\
\delta J_{i} & =i \theta^{a} T^{a} J_{i} & & \delta J_{a}^{+}=f_{a b c} \theta^{b} J^{c+},
\end{aligned}
$$

then the thermal mean values of corresponding fields are given by

$$
\begin{aligned}
& \frac{\delta W_{\beta}}{\delta \bar{\eta}(x)}=\langle\boldsymbol{\Psi}(x)\rangle_{\beta}=\psi(x) ; \quad \frac{\delta W_{\beta}}{\delta \eta(x)}=\langle\bar{\Psi}(x)\rangle_{\beta}=\bar{\psi}(x), \\
& \frac{\delta W_{\beta}}{\delta J_{i}(x)}=\left\langle\boldsymbol{\Phi}_{i}(x)\right\rangle_{\beta}=\phi_{i}(x) ; \quad \frac{\delta W_{\beta}}{\delta J^{a \mu}(x)}=\left\langle\mathbf{A}_{\mu}^{a}(x)\right\rangle_{\beta}=A_{\mu}^{a}(x), \\
& \frac{\delta W_{\beta}}{\delta J_{a}^{*}(x)}=\left\langle\boldsymbol{\omega}_{a}(x)\right\rangle_{\beta}=\omega_{a}(x) ; \quad \frac{\delta W_{\beta}}{\delta J_{b}(x)}=\left\langle\boldsymbol{\omega}_{b}^{*}(x)\right\rangle_{\beta}=\omega_{b}^{*}(x) .
\end{aligned}
$$

The thermal propagators of matter (spinor and scalar) gauge and ghost fields are determined by

$$
\begin{aligned}
\frac{\delta^{2} W_{\beta}}{\delta \bar{\eta}(x) \delta \eta(y)} & =S(x, y) ; & & \frac{\delta^{2} W_{\beta}}{\delta J_{i}(x) \delta J_{k}(y)}=\Delta_{i k}(x, y), \\
\frac{\delta^{2} W_{\beta}}{\delta J_{\mu}^{a}(x) \delta J_{\nu}^{b}(y)} & =G_{\mu \nu}^{a b}(x, y) ; & \frac{\delta^{2} W_{\beta}}{\delta J_{a}(x) \delta J_{b}(y)} & =D_{a b}(x, y) .
\end{aligned}
$$

The effective action at finite temperature is defined normally as a Legendre transformation of $W_{\beta}$

$$
\begin{aligned}
\Gamma_{\beta}\left[\psi, \bar{\psi}, \phi, A_{\mu}, \omega, \omega^{*}\right]= & W_{\beta}\left[\eta, \bar{\eta}, J_{i}, J_{\mu}, J_{a}^{\mu}, J_{a}, J_{b}^{*}\right] \\
& -\int d x\left[\bar{\eta} \psi+\bar{\psi} \eta+J_{i} \phi_{i}+A_{\mu} J_{a}^{\mu}+J_{a}^{*} \omega_{a}+J_{b} \omega_{b}^{*}\right] .
\end{aligned}
$$

The stationary condition for physical processes which correspond to vanishing external sources requires

$$
\begin{aligned}
\left.\frac{\delta \Gamma_{\beta}}{\delta \psi}\right|_{\bar{\eta}=0} & =\left.\frac{\delta \Gamma_{\beta}}{\delta \bar{\psi}}\right|_{\eta=0}=0 ;\left.\quad \frac{\delta \Gamma_{\beta}}{\delta \omega_{a}}\right|_{J_{a}^{*}=0}=\left.\frac{\delta \Gamma_{\beta}}{\delta \omega_{a}^{*}}\right|_{J_{a}=0}=0 \\
\left.\frac{\delta \Gamma_{\beta}}{\delta \phi_{i}}\right|_{J_{i}=0} & =J_{i}-\bar{\psi} g_{i} \psi-\left(D_{\mu}-i \mu \delta_{\mu 0}\right)\left(D^{\mu}-i \mu \delta^{\mu 0}\right) \phi_{i}-m^{2} \phi_{i}-2 \lambda \phi_{i}\left(\phi_{k} \phi_{k}\right)=0 \\
\left.\frac{\delta \Gamma_{\beta}}{\delta A_{\mu}^{a}}\right|_{J_{a}^{\mu}=0} & =J_{\mu}^{a}-\bar{\psi} \gamma_{\mu} T^{a} \psi+\partial^{\nu} F_{\nu \mu}^{a}-f^{a b c} A_{b \nu} F_{c}^{\nu \mu}+\frac{1}{2} T^{a} A_{b \mu} T^{b} \phi_{i}^{2}=0
\end{aligned}
$$

It is shown [7] that the effective action in which the replacement (10)-(12) have been manifested just is original effective action $\Gamma_{\beta}\left[\psi, \bar{\psi}, \phi, A_{\mu}, \omega, \omega^{*}\right]$ evaluated at mean values $A_{\mu}, \psi, \phi$ and $\omega, \omega^{*}$. It is very important to realize that under the formal combined gauge transformation (10)-(12) the effective action is a gauge invariant functional if no external lines $A_{\mu}^{\prime}, \Psi^{\prime}, \Phi^{\prime}$. 
a)
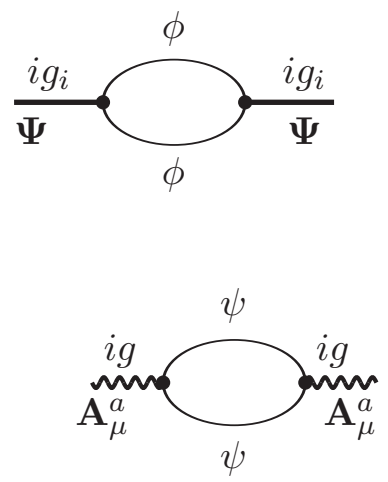

b)

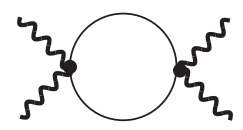

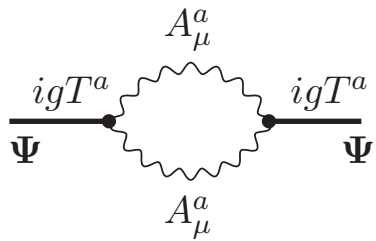
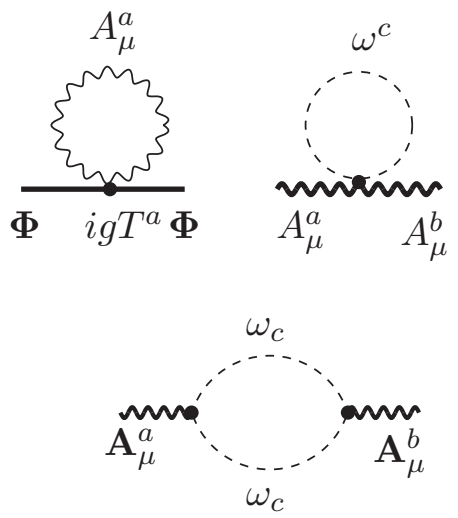
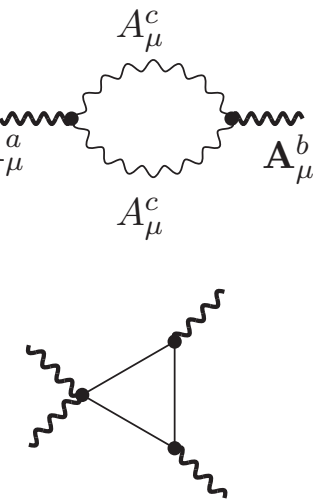

$\mathbf{A}_{\mu}^{a}$

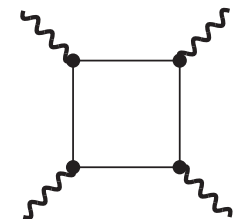

Fig. 1. One loop graphs for background fields which are represented by bold external lines.

a) The internal lines correspond to quantum fields.

b) Three last diagrams are quadratic contribution in background field $A_{\mu}^{a}$

Now we consider the effective action in background field for which $\mathbf{A}_{\mu}^{a}$ and $\boldsymbol{\Phi}_{i}$ are constant, and $\boldsymbol{\Psi}=\overline{\boldsymbol{\Psi}}=\boldsymbol{\omega}=\boldsymbol{\omega}^{*}=0$. For such a background the modified Lagrangian is given by (19) - (24). The effective action is calculated from the part of the action that is quadratic in quantum fields $A_{\mu}^{\prime}, \Phi^{\prime}, \Psi^{\prime}$ and $\omega^{\prime}, \omega^{\prime *}$ over which one integrated

$$
\begin{aligned}
I_{\text {quad }}= & \int d x L_{\text {quad }}=\int d x\left[-\frac{1}{4}\left(\bar{D}_{\mu} A_{\nu}^{\prime a}-\bar{D}_{\nu} A_{\mu}^{\prime a}\right)^{2}-\frac{1}{4} F_{\mu \nu}^{a} f_{a b c} A_{\mu}^{\prime b} A_{\nu}^{\prime c}\right] \\
& -\int d x \bar{\Psi}^{\prime}\left(\gamma^{\mu} D_{\mu}+\mu \gamma^{0}+M+g_{i} \phi_{i}^{\prime}\right) \Psi^{\prime} \\
& +\int d x\left[\frac{1}{2}\left(D_{\mu} \phi_{i}^{\prime} D^{\mu} \phi_{i}^{\prime}-M_{i j}^{2} \phi_{i}^{\prime 2}\right)-\frac{\lambda}{4} \phi_{i}^{\prime 4}\right] \\
& -\int d x\left[\frac{1}{2 \xi}\left(\bar{D}_{\mu} A_{\nu}^{\prime a}\right)^{2}+\left(\bar{D}_{\mu} \omega_{a}^{\prime}\right)\left(\bar{D}_{\mu} \omega_{a}^{\prime}\right)\right] \\
= & \frac{1}{2} \int d x d y A_{\mu}^{\prime a}(x) \mathfrak{D}_{\mu \nu}^{a b}(x, y) A_{\nu}^{\prime b}(y)-\int d x d y \Psi^{\prime}(x) \mathfrak{D}_{a b}(x, y) \Psi^{\prime}(y) \\
& +\frac{1}{2} \int d x d y \phi_{i}^{\prime}(x) \mathfrak{D}_{i k}(x, y) \phi_{k}^{\prime}(y)-\int d x d y \omega_{a}^{\prime *}(x) \mathfrak{D}_{a b}(x, y) \omega_{b}^{\prime}(y)
\end{aligned}
$$


By using the Fourier transformation $\mathfrak{D}(k)=\int d x e^{i k(x-y)} \mathfrak{D}(x-y)$ the matrices in (44) are given by

$$
\begin{aligned}
\mathfrak{D}_{\mu \nu}^{a b}(k)= & g_{\mu \nu}\left[\left(-i k_{\rho} \delta_{c a}+f_{c d a} A_{\rho}^{d}\right)\left(-i k^{\rho} \delta_{c b}+f_{c e b} A_{\rho}^{e}\right)\right. \\
& \left.-\left(-i k_{\nu} \delta_{c a}+f_{c d a} A_{\nu}^{d}\right)\left(-i k_{\mu} \delta_{c b}+f_{c e b} A_{\mu}^{e}\right)+F_{\mu \nu}^{c} f_{c a b}\right] \\
& +g_{\mu \nu} \delta_{i j} \Phi_{i}(k) \Phi_{j}(k) T^{a} T^{b}+\epsilon \text { terms }
\end{aligned}
$$

with $F_{\mu \nu}^{a}=f_{a b c} A_{\mu}^{b} A_{\nu}^{c}$.

$$
\begin{aligned}
\mathfrak{D}(k) & =\left(-i \not k-i T^{a} \mathbb{A}_{\mu}^{a}+M+\mu \gamma_{0}\right)+\epsilon \text { terms, } \\
\mathfrak{D}_{a b}(k) & =\left(-i k_{\rho} \delta_{c a}+f_{c d a} A_{d \rho}\right)\left(i k^{\rho} \delta_{c b}+f_{c e b} A_{e}^{\rho}\right)+\epsilon \text { terms, } \\
\mathfrak{D}_{i j}(k) & =\left(-i k_{\mu}-i \mu-i T_{a} A_{\mu}^{a}\right)_{i}\left(i k_{\mu}+i \mu+i T_{a} A_{\mu}^{a}\right)_{j}-m_{i j}^{2}-\frac{\lambda}{2} \phi_{i} \phi_{j}+\epsilon \text { terms, }
\end{aligned}
$$

In momentum representation, the effective action takes the general form

$$
\begin{aligned}
& \Gamma_{\beta}\left[\psi, \bar{\psi}, \phi, A_{\mu}, \omega, \omega^{*}\right]=I\left[\psi, \bar{\psi}, \phi, A_{\mu}, \omega, \omega^{*}\right]-\frac{i}{2} \operatorname{Tr} \ln G_{\mu \nu}^{a b}(k) \\
& +i \operatorname{Tr} \ln S(k)-\frac{i}{2} \operatorname{Tr} \ln \Delta_{i j}(k)+i \operatorname{Tr} \ln D_{a b}(k)+\sum_{n=2}^{\infty} n \text { loops } 1 P I
\end{aligned}
$$

where the action $I\left[\psi, \bar{\psi}, \phi, A_{\mu}, \omega, \omega^{*}\right]$ is given in (44) - (48). The Trace, the logarithm are taken in functional sense, and the free propagators are given by

$$
\begin{array}{rlrl}
S^{-1}(k) & =k-M-i \epsilon ; & M & =g \nu, \\
\Delta_{i j}^{-1}(k) & =\delta_{i j} k^{2}-M_{i j}^{2}-i \epsilon ; & M_{i j}^{2}=\left(\mu^{2}-m^{2}\right) \delta_{i j}-\frac{\lambda}{2} \phi_{i} \phi_{j}, \\
{\left[G_{0 a b}(k)\right]_{\mu \nu}^{-1}=\left(M_{a b}^{2}-k^{2} \delta_{a b}\right)\left[\frac{k_{\mu} k_{\nu}}{k^{2}}-g_{\mu \nu}\right]+\left[\delta_{a b} \frac{k^{2}}{\xi}-M_{a b}^{2}\right] \frac{k_{\mu} k_{\nu}}{k^{2}},} \\
D_{0 a b}^{-1}(k) & =\delta_{a b}\left(k^{2}-i \epsilon\right), & M_{a b}=\frac{1}{2} \delta_{a b} g \nu .
\end{array}
$$

The higher loop graphs for background fields are represented in Fig. 2.

\section{ONE LOOP FREE ENERGY DENSITY AT $T \neq 0$}

The symmetry is broken if Eqs. (40) - (41) has non-vanishing solutions $A_{\mu} \neq 0, \phi \neq$ 0 . For $\left\langle\mathbf{A}_{\mu}\right\rangle_{\beta}=\delta_{0 \mu} A_{\mu}^{0},\langle\boldsymbol{\Phi}\rangle=\phi_{0}$ the effective potential is defined by

$$
V_{\beta}=-\frac{\Gamma_{\beta}}{\beta \int d x}
$$

It is just thermal free energy density, which concerns with the restoration of symmetry at $T \neq 0$. 


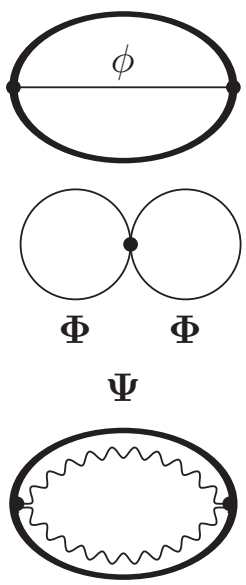

$\Psi$

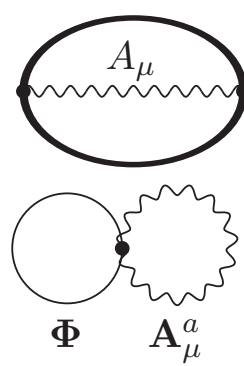

$\Phi$

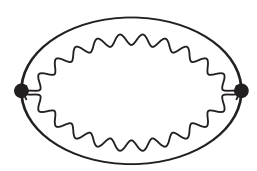

$\Phi$

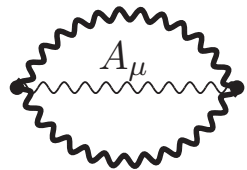

a)

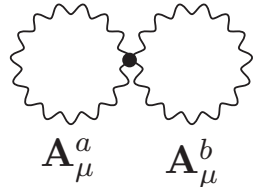

$\mathbf{A}_{\mu}$

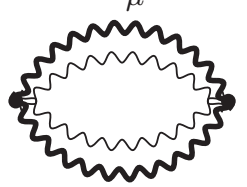

$\mathbf{A}_{\mu}$

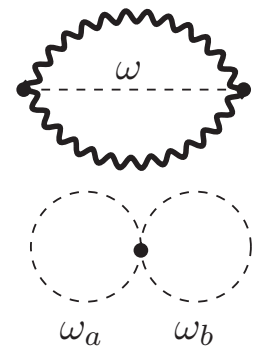

$\mathbf{A}_{\mu}$

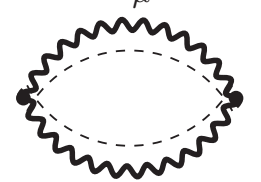

$\mathbf{A}_{\mu}$

Fig. 2. Higher loop graphs for background field gauge: a) Two loop graphs; b)

Three loop graphs

Starting from (49) and (54) the one loop thermal effective potential for the background fields in momentum space reads

$$
V_{\beta}^{1 \text { loop }}=-\int \frac{d^{4} k}{(2 \pi)^{4}}\left[i \operatorname{trln} S(k)-\frac{1}{2} \operatorname{trln} \Delta_{i j}(k)-\frac{1}{2} \operatorname{trln} G_{\mu \nu}(k)+i \operatorname{trln} D_{a b}(k)\right]
$$

here "tr" denotes usual traces of finite matrices.

The proper way to regularize the theory at finite temperature in $d=4-2 \epsilon$ dimensions is apply the "imagine time" formalism, where all four momenta are Euclidean with discrete Matsubara frequencies $k_{4}=i \omega_{n}$

i.e.

$$
\omega_{n}= \begin{cases}2 n \pi T & \text { for bosons } \\ (2 n+1) \pi T & \text { for fermions }\end{cases}
$$

$$
\int \frac{d^{4} k}{(2 \pi)^{4}}=i \int \frac{d^{4} k_{E}}{(2 \pi)^{4}} \rightarrow i T \sum_{n=-\infty}^{\infty} \int \frac{d^{3} k}{(2 \pi)^{3}}=\sum_{k} \text {. }
$$

In $d=4-2 \epsilon$ dimensions it is replaced by

$$
\sum_{k} \rightarrow \eta^{2 \epsilon} T \sum_{n=-\infty}^{n=+\infty} \frac{d^{3-2 \epsilon}}{(2 \pi)^{3-2 \epsilon}}
$$

and then perform $\epsilon \rightarrow 0$ limit. Here $\eta$ is renormalization scale.

From (44) - (49) we arrive at the expression for the effective potential

$$
\begin{aligned}
V_{\beta}= & V_{c l}-\sum_{k}\left[\ln \left(k^{2}+M^{2}\right)-\ln \left(k^{2}+M_{i j}^{2}\right)-\ln \left(k^{2}+M_{a b}^{2}\right)\right] \\
& +i g^{2} J\left(\frac{11}{12} N-\frac{1}{6} N_{F}+\frac{1}{12} N_{B}\right) \int d x F_{\mu \nu}^{a} F^{a \mu \nu}+\frac{i}{8} g^{2} K+\frac{i}{2} g_{i} P
\end{aligned}
$$


where $N_{F}$ and $N_{B}$ are fermionic and bosonic number in defining representation. The third term calculated from (45) -(48) is one loop contribution in the background gauge field. Two last terms are bosonic contribution to $V_{\beta}$ and the fermionic self energy. Here $J, K, P$ are divergent integrals.

$$
\begin{aligned}
J & =\int \frac{d^{4} k}{(2 \pi)^{4}} \frac{1}{\left(k^{2}-i \epsilon\right)^{2}} \rightarrow \sum_{k} \frac{1}{\left(k^{2}\right)^{2}} \\
K & =\int \frac{d^{4} k}{(2 \pi)^{4}} \frac{1}{\left(k^{2}-m^{2}-i \epsilon\right)^{2}} \rightarrow \sum_{k} \frac{1}{\left(k^{2}+m^{2}\right)^{2}} \\
P & =\sum_{k} \sum_{p} \frac{1}{\left(p^{2}+M_{1}^{2}\right)\left(k^{2}+M_{2}^{2}\right)\left[(k+p)^{2}+m_{3}^{2}\right]} .
\end{aligned}
$$

For massless bosons and ghosts, we have

$$
\begin{aligned}
J & =\int \frac{d^{4} k}{(2 \pi)^{4}} \frac{1}{\left(k^{2}-i \epsilon\right)^{2}} \rightarrow \sum_{k} \frac{1}{k^{2}} \\
& =\eta^{2 \epsilon} \int \frac{d^{3-2 \epsilon} k}{(2 \pi)^{3-2 \epsilon}} \frac{1}{k^{2}}+2 \eta^{2 \epsilon} T \sum_{n=1}^{\infty} \int \frac{d^{3-2 \epsilon} k}{(2 \pi)^{3-2 \epsilon}} \frac{1}{\left[(2 \pi n T)^{2}+\mathbf{k}^{2}\right]^{2}} \\
& =\frac{1}{(4 \pi)^{2}}\left[\frac{1}{\epsilon}+2 \ln \frac{\bar{\eta}}{4 \pi T}+2 \gamma_{E}\right]+0(\epsilon),
\end{aligned}
$$

where $\eta^{2}=\frac{e^{\gamma} E \bar{\eta}^{2}}{4 \pi}$ (P.Arnold and C.Zhai, $1994[8]$ ).

In the case $m \neq 0$, the integral contributed to one loop potential takes the form

$$
I(m)=\int \frac{d^{4} k}{(2 \pi)^{4}} \frac{1}{k^{2}-m^{2}-i \epsilon} \rightarrow \sum_{k} \frac{1}{k^{2}+m^{2}} .
$$

Using the well - known result [9]

$$
\begin{aligned}
\frac{1}{2} \operatorname{Tr} \ln \left(k^{2}-m^{2}\right) & \equiv \frac{1}{2} \sum_{k} \ln \left[-(\pi n T)^{2}-\mathbf{k}^{2}-m^{2}\right] \\
& \simeq-\frac{\pi^{2} T^{4}}{90}+\frac{m^{2} T^{2}}{24}-\frac{m^{3} T}{12 \pi}-\frac{m^{4}}{64 \pi^{2}} C,
\end{aligned}
$$

where $C=\frac{1}{\epsilon}+2 \ln \frac{\bar{\eta}}{4 \pi T}+2 \gamma_{E}$, we can evaluate the integrand at high temperature.

$$
I(m) \equiv \frac{1}{m} \frac{\partial}{\partial m}\left[\frac{1}{2} \operatorname{Tr} \ln \left(k^{2}-m^{2}\right)\right]=\frac{T^{2}}{12}-\frac{m T}{4 \pi}+\frac{m^{2} C}{(4 \pi)^{2}} .
$$

It is straightforward to extend this result to $(61), K(m)=I^{2}(m)$, where $m^{2}$ is replaced by thermal mass $\mathfrak{M}^{2}$

$$
\begin{aligned}
K(\mathfrak{M})= & \text { const }-\frac{g^{2}}{24 \pi} \mathfrak{M} T^{3}+\frac{1}{(4 \pi)^{2}} g^{2} \mathfrak{M}^{2} T^{2} \\
& -\frac{1}{(4 \pi)^{2}} g^{2} \mathfrak{M}^{2} T^{2}\left(\frac{1}{\epsilon}+2 \ln \frac{\bar{\eta}}{4 \pi T}+2 \gamma_{E}\right) .
\end{aligned}
$$


Thus, there is $1 / \epsilon$ term, i.e UV divergence in both $J, K$. The $P$ integral in (62) corresponds to the sun diagram $[10]$

$$
P^{b}\left(m_{1}, m_{2}, m_{3}\right)=\quad=\frac{T^{2}}{(4 \pi)^{2}}\left[\frac{1}{4 \epsilon}+\ln \frac{\bar{\eta}}{m_{1}+m_{2}+m_{3}}+\frac{1}{2}\right]+0(m, \epsilon) .
$$

When fermions are included, it is vanished

$$
P^{f}\left(M_{1}, M_{2}, m\right)=\quad=0(\epsilon) .
$$

The total temperature dependent part of the loop effective potential is the sum of terms (63)-(68). The one loop thermal free energy density takes the form

$$
\begin{aligned}
V_{\beta}= & -\frac{1}{2}\left(\mu^{2}-m^{2}\right) \phi^{2}+\frac{\lambda}{4} \phi^{4}-\frac{1}{2} \delta_{a b} M_{a b}^{2} A_{0 \mu}^{2}-\frac{\pi^{2} T^{4}}{90}\left(N_{B}+\frac{7}{8} N_{F}\right) \\
& +\frac{T^{2}}{24}\left\{\left(\mu^{2}-m^{2}-\frac{\lambda}{2} \phi^{2}\right)+3 \operatorname{Tr} M_{a b}^{2}+\frac{1}{2} \operatorname{Tr}\left[\gamma_{0}\left(M+\mu \gamma_{0}\right) \gamma_{0}\left(M+\mu \gamma_{0}\right)\right]\right\} \\
& -\frac{T}{12 \pi}\left(\mathfrak{M}^{3}+\delta_{a b} M_{a b}^{3}\right)-\frac{g^{2} T^{3}}{48 \times 4 \pi}\left(\mathfrak{M}+\delta_{a b} M_{a b}+2 M\right) \\
& +\frac{g^{2}}{(4 \pi)^{2}}\left(\frac{11}{12} N-\frac{1}{6} N_{F}+\frac{1}{12} N_{B}\right)\left(\frac{1}{\epsilon}-2 \ln \frac{\bar{\eta}}{4 \pi T}+2 \gamma_{E}\right) \int d x F_{\mu \nu}^{a} F^{a \mu \nu} .
\end{aligned}
$$

Hence, it is easily to derive the thermal masses, e.g in the first approximation the scalar thermal mass is

$$
\mathfrak{M}^{2}=\left(\mu^{2}-m^{2}\right)+\frac{\lambda}{24} T^{2}-\frac{\lambda}{2} \phi^{2} .
$$

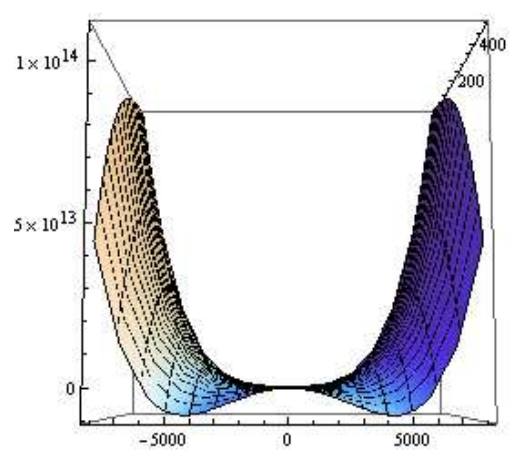

a)

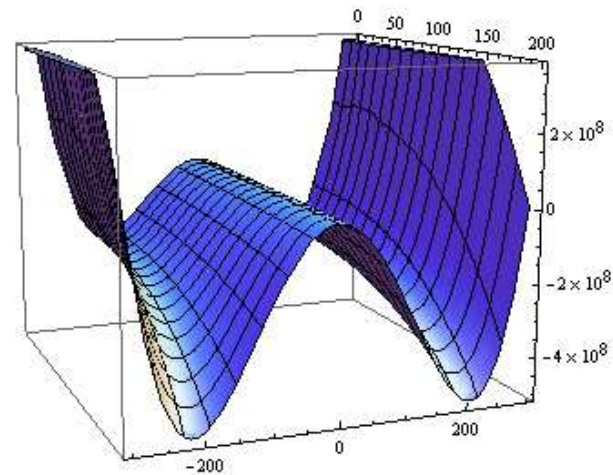

b)

Fig. 3. Plot for the effective potential described in Eq.(69) with $g=10 \mathrm{GeV}$, $\lambda=0.1, T=0 \div 200 \mathrm{MeV}$. a) $\mu / m=0.5$; b) $\mu / m=1.2$ 
Finally, the infinite factor $L_{A}$ is determined from (27), (59) and (63)

$$
\begin{aligned}
L_{A} & =4 i g^{2} J\left(\frac{11}{12} N-\frac{1}{6} N_{F}+\frac{1}{12} N_{B}\right) \\
& =-\frac{g^{2}}{(2 \pi)^{2}}\left(\frac{11}{12} N-\frac{1}{6} N_{F}+\frac{1}{12} N_{B}\right)\left(\frac{1}{\epsilon}+2 \ln \frac{\bar{\eta}}{4 \pi T}+2 \gamma_{E}\right)+0\left(g^{4}\right) .
\end{aligned}
$$

The renormalized coupling $g_{R}$ in (37) is given by

$$
g_{R}=g\left[1+\frac{g^{2}}{4 \pi^{2}}\left(\frac{11}{12} N-\frac{1}{6} N_{F}+\frac{1}{12} N_{B}\right)\left(\frac{1}{\epsilon}+2 \ln \frac{\bar{\eta}}{4 \pi T}+2 \gamma_{E}\right)\right]+0\left(g^{4}\right) .
$$

Note that in non-Abelian theory, the physical coupling $g_{R}$ increases due to quantum corrections at high temperature, as well as at zero temperature and chemical potential. It is just the difference with the Abelian theories.

\section{DISCUSSION AND CONCLUSION}

In the above sections we have presented in detail the background field method and its effective action for the non-Abelian theory at finite temperature. The renormalization for the background field gauge is manifested at $T \neq 0$. Much interest is focused only on the leading correction to the one loop free energy density. Hence the numerical computation and the cosmological phase transition would be investigated in our next work. Furthermore, the non-Abelian Higgs mechanism in non-perturbative sector [11] or the Kaon condensate at high temperature in the early Universe [12] could be likely considered by this way.

\section{ACKNOWLEDGMENT}

The author would like to thank Prof. Tran Huu Phat for helpful suggestion of this problem.

\section{REFERENCES}

[1] J. I. Kapusta, Finite Temperature Field Theory, Cambridge University Press, Cambridge (1989).

[2] L. F. Abbott and S-Y-Pi, Inflationary Cosmology, World Scientific, Singapore (1986).

[3] R. Jackiw, G. Amelia - Camelia, Field Theoretical Background for Thermal Physics, Third workshop on Thermal Field Theories, Banff, Canada, 1993.

[4] B. S. De Witt, A Gauge Invariant Effective Action, in Quantum Gravity II, Oxford: Oxford University Press, 1981.

[5] S. Hashimoto, J. Kodaira, Y. Yasmi and K. Saraki, Phys. Rev. D50 (1994) 11.

[6] S. Weinberg, The Quantum Theory of Fields II, Cambridge University Press, Cambridge (1986).

[7] S. Pokorski, Gauge Field Theory, Cambridge University Press, Cambridge,1987.

[8] P. Arnold and C. Zhai, Phys. Rev. D49 (1994) 2740.

[9] L. Doland and R. Jackiw, Phys. Rev. D9 (1974) 3320.

[10] P. Arnold and D. Espinosa, Phys. Rev. D47 (1993) 3546.

[11] E. Braaten, Phys. Rev. Lett. 14 (1995) 164.

[12] V. A. Miransky and I.Shokovy, Phys. Lett. 88 (2002) 111601. 